\title{
Unusual Rearrangements of Radical Cations: The Role of Vibronic Coupling
}

\author{
Thomas Bally*
}

\begin{abstract}
Radical cations often undergo very unexpected rearrangements. Three examples of such rearrangements are given, and it is shown how vibronic coupling between the ground and low-lying excited states may cause certain bonds that are quite solid in the neutral molecules to become so weak that they break spontaneously, even though the bond order does not change (or changes very little) on ionization. In radical cations where spin and charge are delocalized over two similar halves, vibronic coupling can lead to localization of spin and charge, which may greatly affect the reactivity. Finally, it is shown how vibronic coupling can lead to avoidance of state crossings, a feature that appears very frequently in rearrangements of radical cations.
\end{abstract}

Keywords: Radical cations · Vibronic coupling

\section{Introduction}

Radical cation chemistry often differs from the reactivity of the neutral molecules in that very surprising and unusual thermal or photochemical rearrangements or fragmentations can occur. We have investigated many of these reactions over the course of time. In this paper I will mainly focus on the first of the startling rearrangements shown in Scheme 1, and on the reason why it takes such a different course than it does in the neutral precursors. I will use this example to illustrate how synergy between experimental and computational chemistry can lead to the solution of problems which could most probably not have been solved by either approach alone. Then I will describe more briefly the other two examples, and what they taught us.

All three of the rearrangements in Scheme 1 involve distortions that require high amounts of energy in the neutral precursors, but become very facile, or even spontaneous, in the radical cations. As it turns out, the reason is that radical cations often have very low-lying excited states that may couple to the ground states upon certain distortions, and thus flatten or even bend down the ground state surfaces, a phenomenon known as vibronic coupling.

As will be explained below, the consequence of vibronic coupling is that bonds which are quite solid in a neutral molecule may become so weak in the radical cations that they undergo very surprising and al-

\footnotetext{
${ }^{\star}$ Correspondence: Prof. Dr. T. Bally

Department of Chemistry

University of Fribourg

Chemin du Musée 9

$\mathrm{CH}-1700$ Fribourg, Switzerland

E-mail: Thomas.Bally@unifr.ch
}

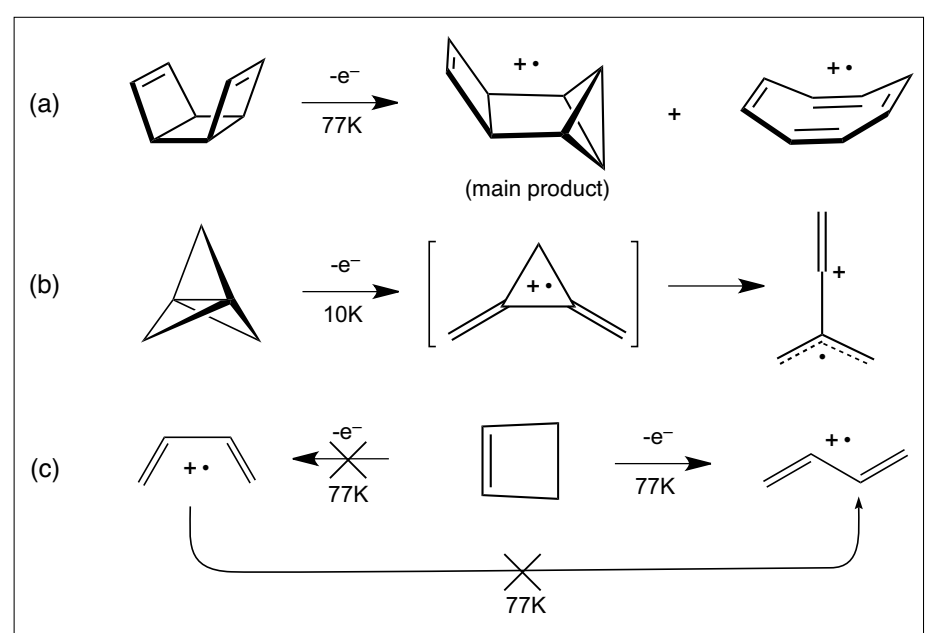

Scheme 1. Three examples of surprising radical cation rearrangements. ${ }^{[1]}$

most barrierless rearrangements. We have investigated several such processes in detail by means of quantum chemical calculations, which allowed us to explain why they occur in the way they do. The techniques that were used to arrive at the spectra shown in some of the following Figures have been described in detail. ${ }^{[2]}$

\section{The Case of syn-Tricyclo[4.2.0.0 2,5]} octa-3,7-diene (syn-TOD) Radical Cation[1a]

The syn-dimer of cyclobutadiene, synTOD, decays upon heating to $>100{ }^{\circ} \mathrm{C}$, by two consecutive cyclobutene-butadiene ring openings to cyclooctatetraene (COT) ${ }^{[3]}$ Its radical cation gives the same compound, but only as a side product and at much lower temperature. Upon radiolysis of syn-TOD at $77 \mathrm{~K}$ in a Freon glass, the spectrum shows, next to the prominent band of COT $^{\circ+}$ peaking at $505 \mathrm{~nm}$, another broad band peaking at $c a .820 \mathrm{~nm}$ which eventually turned out to be identi- cal to the one obtained by radiolysis of tetracyclo[4.2.0.0 $\left.0^{2,4} \cdot 0^{3,5}\right]$-oct-7-ene (TCO) under the same conditions (see Fig. 1).

Although TCO is a valence isomer of TOD we had no clue how this rearrangement can take place in the radical cations, and whether any intermediates are involved on the way, which could perhaps be stabilized and detected below $77 \mathrm{~K}$. Thus we asked colleagues of ours from Poland, who are equipped to do pulse radiolysis at lower temperatures, to repeat the experiment (Fig. 2). To our surprise, they found a completely different spectrum at $30 \mathrm{~K}$, marked by a very intense band peaking at $545 \mathrm{~nm}$ which decayed on warming to 77 $\mathrm{K}$ to form the (much weaker) NIR band which we had also observed ( $c f$. Fig. 2).

On further warming to $90 \mathrm{~K}$ that band disappeared again (Fig. 2) and was replaced by a spectrum which we identified as being that of the radical cation of bicyclo[4.2.0]octa-2,4,7-triene, BOD ${ }^{\circ+}$ (the same spectrum was also obtained on photolysis of the NIR band which we later assigned to $\mathrm{TCO}^{*+}$ ). We would have expected this spe- 

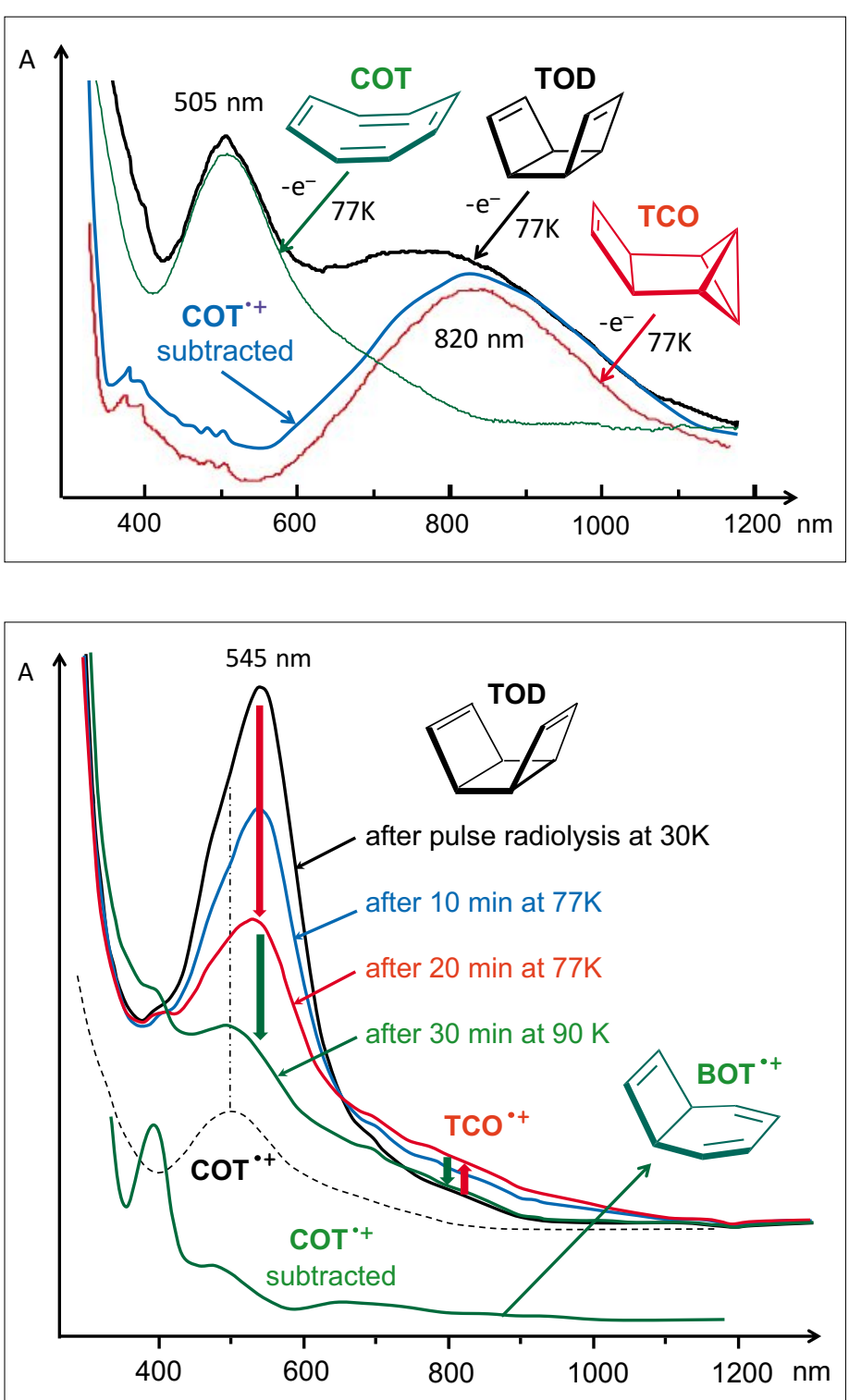

Fig. 1. Spectrum obtained on radiolysis of TOD in a Freon glass at $77 \mathrm{~K}$ (black line); spectra obtained from COT (green line) and from TCO (red line) under the same conditions; difference between the black and the green spectrum (blue line).

Fig. 2. Spectrum obtained after electron pulse radiolysis of TOD at $30 \mathrm{~K}$ in a methylcyclohexane/ butyl chloride glass (black line); decay of this spectrum on warming to $77 \mathrm{~K}$ (blue and purple line), and after additional warming to $90 \mathrm{~K}$ (red line). Spectrum of COT $^{0+}$ (dashed line, cf. Fig. 2), and spectrum obtained after subtracting the dashed black spectrum from the red one (green line). The latter spectrum could be assigned unambiguously to BOT॰+.
For the purpose of this paper I have rerun all the calculations with a modern functional (M06-2X), and the relative energies are based on single-point $\operatorname{CCSD}(\mathrm{T}) / \mathrm{cc}-$ pVTZ calculations, which was not possible at the time when we did the original work. ${ }^{[1 a]}$

syn-TOD ${ }^{+}$has a totally symmetric ground state $\left({ }^{2} \mathrm{~A}_{1}\right)$ which, according to the photoelectron spectrum of TOD, ${ }^{[4]}$ is separated from the first excited state $\left({ }^{2} \mathrm{~B}_{1}\right)$, by $c a .7 .5 \mathrm{kcal} / \mathrm{mol}$ at the neutral geometry. If both of these states relax their geometry, while maintaining the $C_{2 v}$ symmetry of the neutral molecule, the ${ }^{2} \mathrm{~A}_{1}$ ground state drops by $c a$. $7.5 \mathrm{kcal} / \mathrm{mol}$ in energy, while the ${ }^{2} \mathrm{~B}_{1}$ excited state drops by almost twice as much, and thus falls $c a .3 \mathrm{kcal} / \mathrm{mol}$ below the ${ }^{2} \mathrm{~A}_{1}$ ground state (see dashed lines in Fig. 3).

Surprisingly, the ${ }^{2} \mathrm{~A}_{1}$ state turns out to be a saddle point on the M06-2X potential energy surface, a structure which decays spontaneously by breaking one or the other of the lateral bonds in the central cyclobutane moiety $\left(r_{3}\right.$ or $r_{4}$ in Fig. 3) to yield a species which we termed bis(cyclobutenylium), $\mathbf{B C B}^{\cdot+}$. This species distinguishes itself by a so-called charge resonance band (cf. Fig. 4), an electronic transition between the bonding and the antibonding combination of the two allylic non-bonding MOs. Such transitions occur in radical cations that are composed of two interacting parts with the same electronic structure, (for example dimer radical cations ${ }^{[5]}$ ) and they are usually very intense. In fact, CASPT2 excited state calculations predict this transition to occur at $c a$. $490 \mathrm{~nm}$ in $\mathrm{BCB}^{\circ+}$ (with a very high oscillator strength of 0.22 ), in very good accord cies to arise as an intermediate on the way to $\mathrm{COT}^{\circ+}$, but not as a product of the decay of $\mathrm{TCO}^{*+}$ !

To explain these rather unexpected findings, we explored the electronic structure and the potential energy surfaces of syn-TOD ${ }^{*+}$ by quantum chemical calculations. To understand the following Figures, note that different electronic states of radicals can be characterized by their singly occupied molecular orbital (SOMO), which also imposes the symmetry of the state of the radical. For ground states of radical cations, the SOMO is the MO from which an electron was removed on oxidation, and the nodal structure of the SOMO indicates for example which bonds are weakened and which are strengthened in this process. Upon electronic excitation, an electron can be promoted from one of the doubly occupied bonding MOs to the SOMO, or from the SOMO to the LUMO, whereupon a new orbital becomes the SOMO. Thus we will frequently depict SOMOs in the Figures below to show what state of a radical cation we are dealing with.

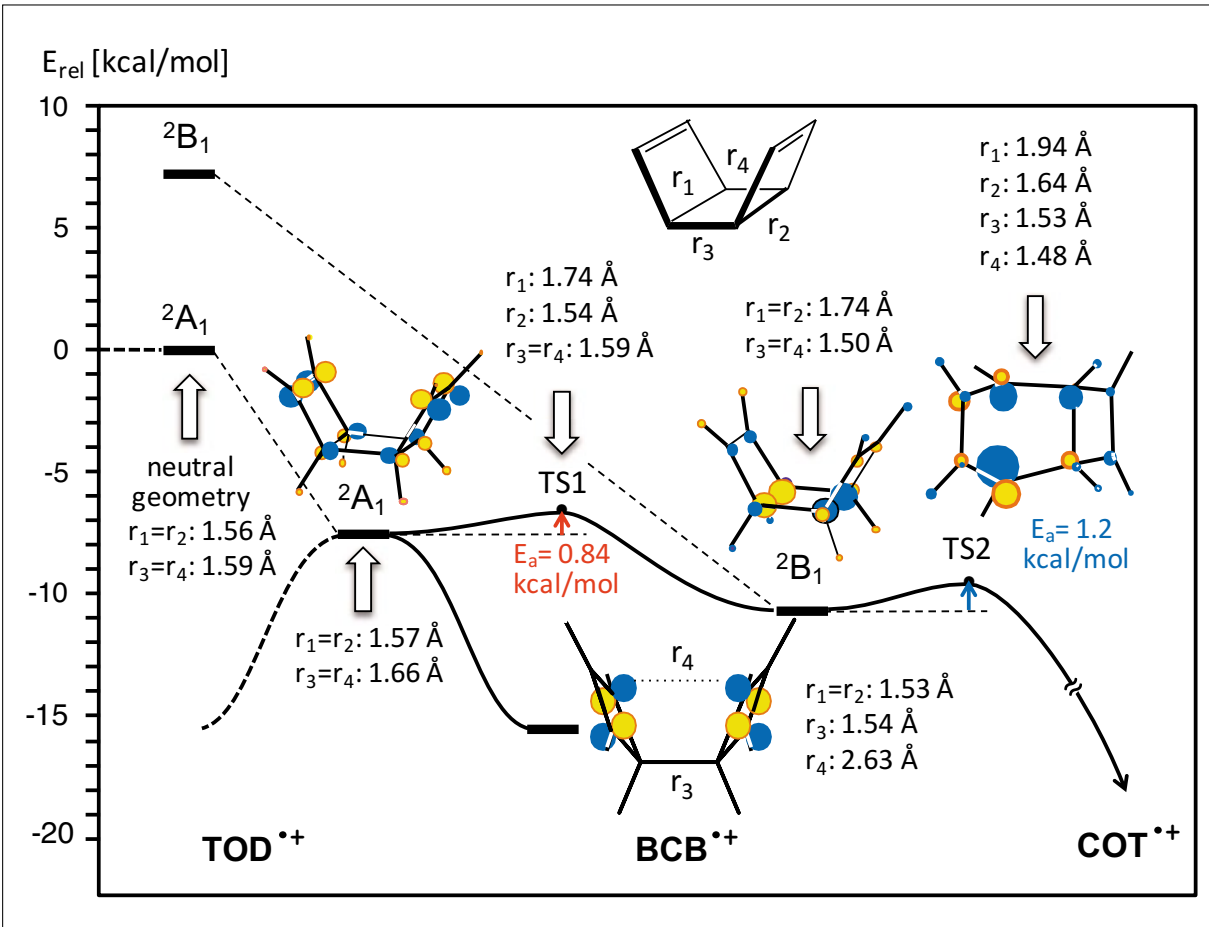

Fig. 3. Decay of syn-TOD upon ionization, according to $\operatorname{CCSD}(\mathrm{T}) / / \mathrm{M} 06-2 \mathrm{X}$ calculations. 
with the strong band observed at $475 \mathrm{~nm}$ after radiolysis at $30 \mathrm{~K}$.

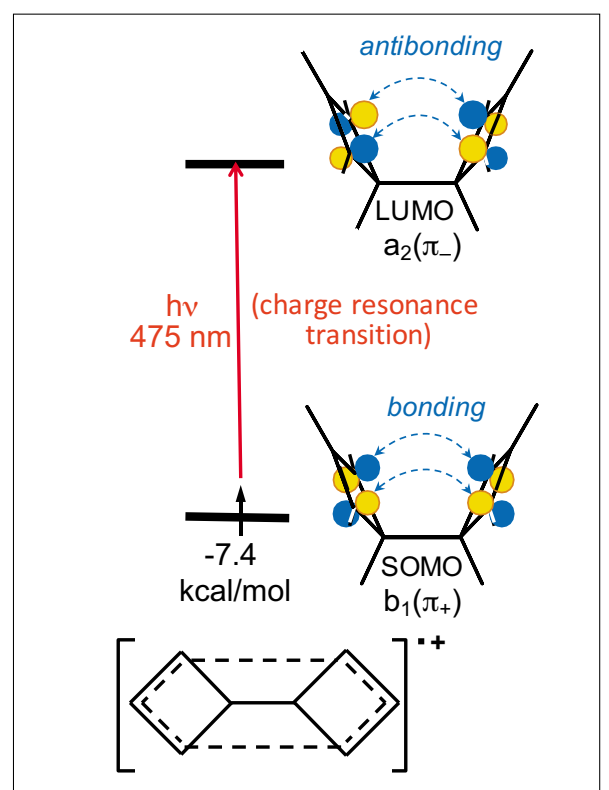

Fig. 4. Electronic structure of $\mathbf{B C B}^{\bullet+}$.

On the other hand, we found that the adjacent bonds in the central cyclobutane moiety of the ${ }^{2} \mathrm{~A}$, state of TOD ${ }^{*+}$ (denoted by $r_{1}$ and $r_{2}$ in Fig. 3) are also quite labile and one of them can be stretched by $0.2 \AA$ at the expense of less than $1 \mathrm{kcal} /$ mol. Thereby the molecule reaches a lowlying transition state (TS1 in Fig. 3) which decays by stretching the opposite bond, whereby it ends up in the potential energy minimum of the ${ }^{2} \mathrm{~B}_{1}$ state. Upon further stretching of this bond, one approaches another very low-lying transition state (TS2), with no symmetry, which we would have expected to decay to $\mathbf{B O T}^{*+}$.

However, a so-called 'intrinsic reaction coordinate' (IRC) calculation, which leads from a transition state (= saddle point) along a potential valley to the closest minimum, showed that the reaction path actually bypasses $\mathbf{B O T}^{\circ+}$ and leads directly to $\mathbf{C O T}^{-+}$. The reason for this is that the TOD $^{\bullet+}\left({ }^{2} \mathrm{~B}_{1}\right) \rightarrow$ BOT $^{*+}$ rearrangement involves a state crossing which must be avoided by a distortion of the molecule that is probably too costly to compete with the direct decay to COT $^{-+}$(see Fig. 5, which also shows that the valence isomerization of $\mathbf{B O T}^{*+}$ to $\mathbf{C O T}^{++}$involves an avoided state crossing, see below).

Thus, the calculations shown in Figs 3 and 5 explain why, after ionization of synTOD, we see a mixture of $\mathbf{B C B}^{\cdot+}$ (where one bond is broken in a barrierless process) and $\mathbf{C O T}^{-+}$, where two bonds are broken with a tiny barrier. But then, dynamic effects could very well cause a fraction of the relaxing TOD $^{++}$to be projected in the direction of this tiny barrier so it can be crossed, even though this process competes with the barrierless decay to $\mathbf{B C B}^{\cdot+}{ }^{\cdot 6]}$

However, what these calculations do not explain is why the bonds in the central cyclobutane moiety of TOD become so weak on ionization that one or even two of them break spontaneously at $30 \mathrm{~K}$. To answer this question we first need to understand the nature of vibronic coupling and the effect it has on the shape of potential surfaces.

Vibronic coupling occurs typically between two electronic states, in our case the ground state $\Psi_{0}$ and an excited state $\Psi_{\mathrm{i}}^{*}$ of a

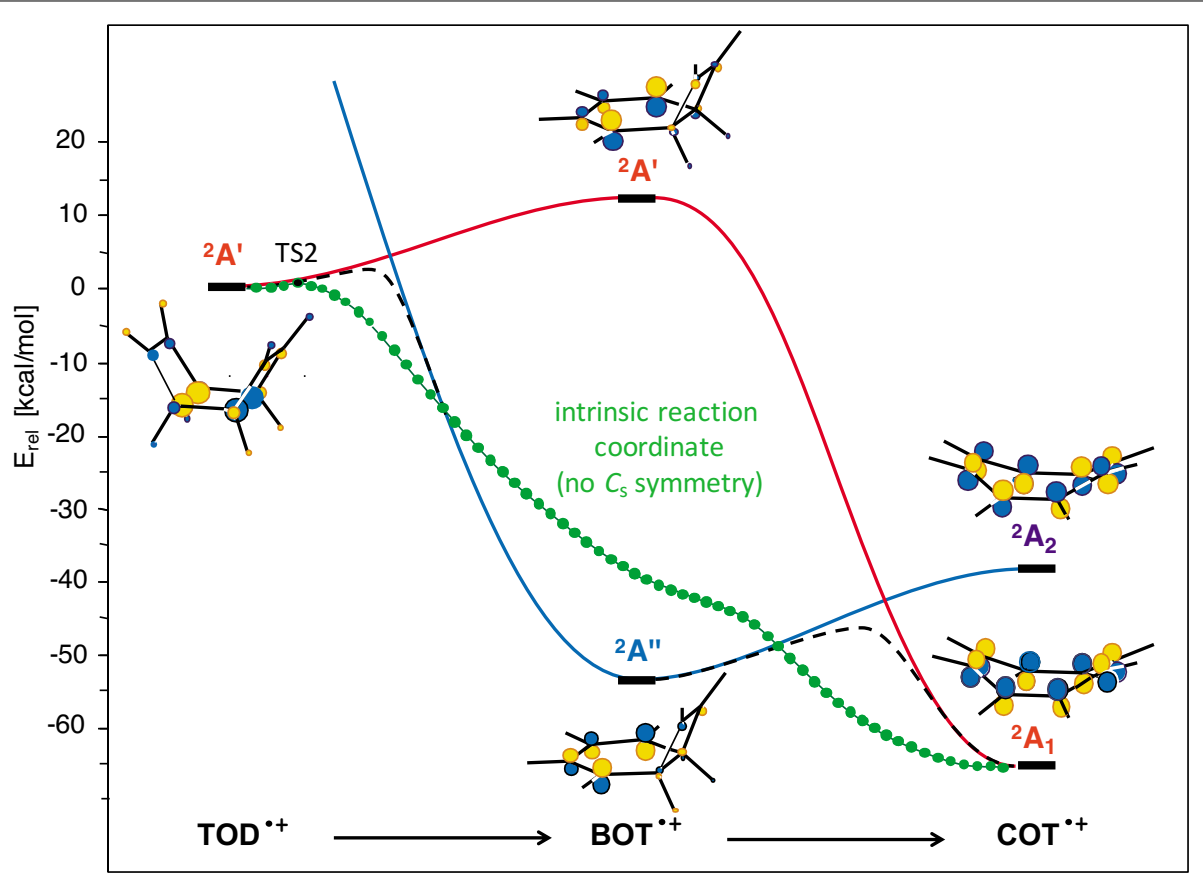

Fig. 5. State correlation diagram in $\mathrm{C}_{\mathrm{S}}$ symmetry for the decay of ${ }^{2} \mathrm{~B}_{1}$ syn-TOD*+ to COT$^{\bullet+}$ (solid red and blue line) via BOT*+, and results of an IRC calculation (green dots) which shows that BOT $^{++}$is bypassed in the process. TS2 is the same as in Fig. 4. molecule which, at a symmetric geometry, cannot interact because the two states are of different symmetry (properly expressed: they belong to different irreducible representations, $\Gamma_{\mathrm{i}}$, within the point group of the molecule).

However, upon distorting the molecule in a way that breaks its geometrical symmetry, the $\Gamma_{\mathrm{i}}$ of the two states may become the same whereupon they can interact. Often such distortions correspond to normal modes of vibration of the molecule which may lead to coupling of two electronic states, which is why the phenomenon is called vibrational-electronic or 'vibronic' coupling. As a consequence of the interaction of the two states, the energy of the lower one is lowered and that of the higher one is raised along the distortion coordinate $q$ (Fig. 6). ${ }^{[7]}$ When the vibronic coupling is strong, the lower potential surface can even bend down to form a double minimum (purple dashed line).

Let us illustrate this interesting feature on the example of TOD $^{-+}$that we have discussed above: Fig. 7 shows the ground state and the first three excited states of TOD ${ }^{*}$, each of them characterized by its SOMO. As the ground state $\Psi_{0}$ of TOD ${ }^{*+}$ is totally symmetric $\left({ }^{2} \mathrm{~A}_{1}\right)$, the distortions $q$ which can induce a mixing of $\Psi_{0}$ with the excited states $\Psi_{\mathrm{i}}^{*}$ must have the same symmetry as the excited states, respectively $($ i.e. $\Gamma(q)=$ $\left.\Gamma\left(\Psi_{\mathrm{i}}^{*}\right)\right)$, as indicated in Fig. 7.

What are the consequences of a distortion which induces a mixing of $\Psi_{0}$ with $\Psi_{\mathrm{i}}^{*}$ depends on three factors: (i) the extent to which the two states mix, (ii) their energy separation and (iii) on the force that opposes the distortion. The first two factors are described by Eqn. (1), the numerator of which measures factor (i) and is called 'derivative coupling'. This is, however, not a numeral, but a vector which indicates the mode of distortion along which $\Psi_{0}$ and $\Psi_{\mathrm{i}}^{*}$ undergo optimal mixing (the length of the vector indicates the extent of coupling between the two states, which depends on the overlap of the two wavefunctions, or, in the case of radicals of the two SOMOs).

extent of vibronic coupling $=\frac{\left\langle\Psi_{0}\left|\frac{\partial}{\partial q}\right| \Psi_{i}\right\rangle}{E_{i}-E_{0}}$

The denominator corresponds to the energy difference between the two states at the symmetric geometry $(q=0)$, i.e. the closer two states are, the stronger will be the vibronic coupling between them (as it is the case with interacting orbitals). Because radical cations often have very low-lying excited states, compared to neutral, closed-shell molecules, vibronic coupling manifests itself much more frequently and extensively in radical cations. 


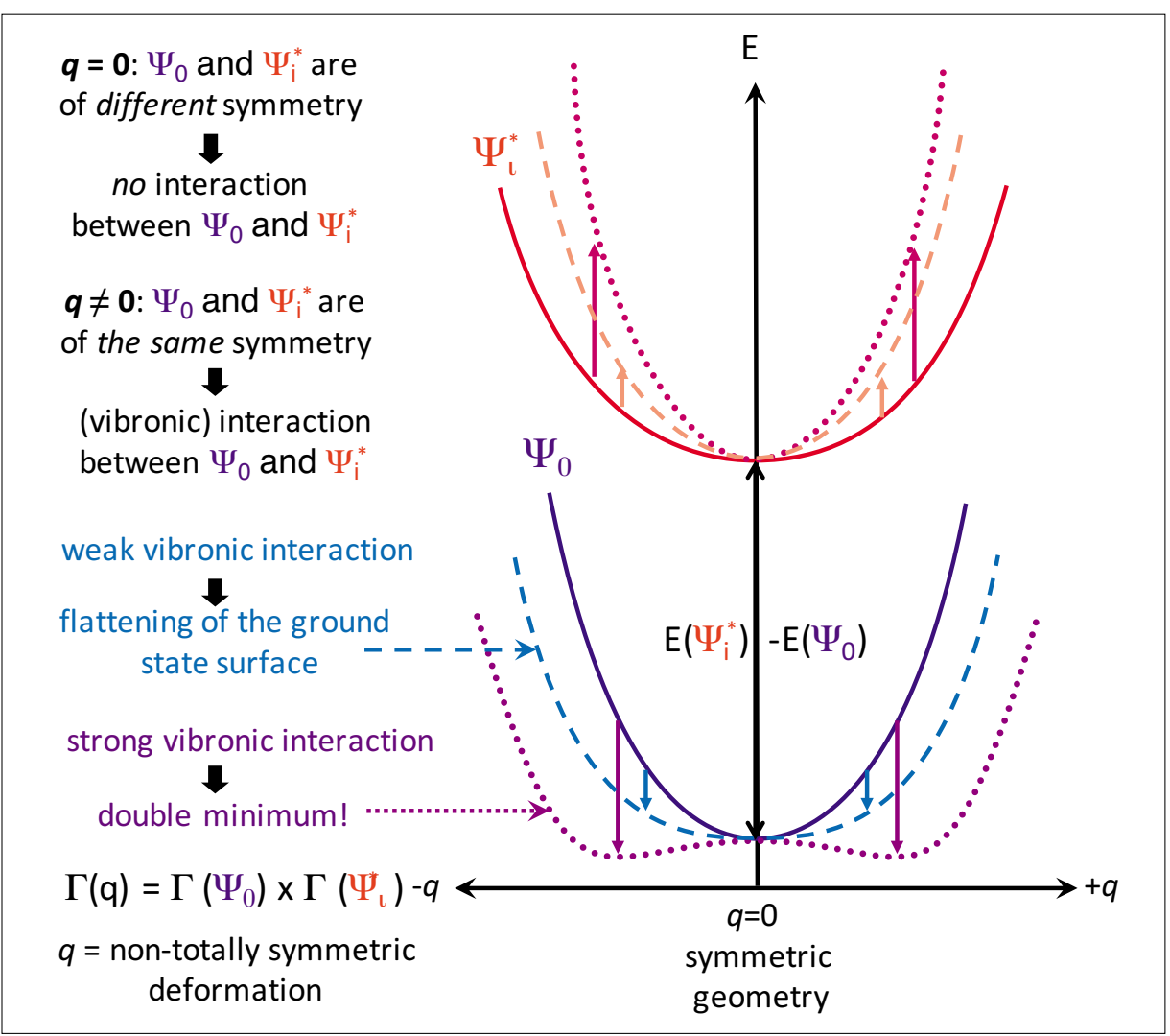

Fig. 6. Pictorial representation of vibronic coupling and its consequences.

These two factors are also illustrated in Fig. 7 for the example of TOD ${ }^{++}$. The energy differences are given in blue according to calculations at the optimized geometry of the ${ }^{2} \mathrm{~A}_{1}$ ground state of TOD $^{\cdot+}$, while the derivative coupling vectors, calculated at the CASSCF level, are shown in red.

Due to the small energy gap, the lowest-lying excited state is often expected to mix most strongly with the ground state. In the ${ }^{2} \mathrm{~A}_{1}$ ground state and the first ${ }^{2} \mathrm{~B}_{1}$ excited state of TOD $^{*+}$, the SOMO corresponds largely to the positive and negative combination, respectively, of the olefinic $\pi-$ MOs $^{[4]}$ (if the ${ }^{2} \mathrm{~A}_{1}$ state of TOD $^{-+}$could be observed, the excitation to this ${ }^{2} \mathrm{~B}_{1}$ state would correspond to a very intense charge resonance transition in the near IR). Hence the mixing of these two states is primarily induced by an antisymmetric stretching of these double bonds, which is indeed associated with a strong derivative coupling ( $c f$. Fig. 7), but as this deformation is also associated with a high force constant (factor iii above), the only consequence of this strong vibronic coupling is that the frequency for the antisymmetric double bond stretching vibration decreases by $c a .200 \mathrm{~cm}^{-1}$.

In the second excited state the SOMO also has $b_{1}$ symmetry but it is largely localized in the central four-membered ring, hence the coupling of the two states must involve an antisymmetric deformation of this ring, along $a b_{1}$ coordinate. As the AOs that constitute the $a_{1}\left(\pi_{+}\right)$and the $\mathrm{b}_{1}(\sigma)-\mathrm{MO}$ are largely orthogonal, their becomes nevertheless very flat as a consequence of the vibronic coupling. This ex- overlap is small and hence the derivative coupling vector is much shorter than constant opposing the deformation of than for the stretching of the double bonds, and hence the surface for the deformation indicated by the derivative coupling vector presses itself chemically in the very low activation barrier for the passage of the ${ }^{2} \mathrm{~A}_{1}\left(\pi^{+}\right)$to the ${ }^{2} \mathrm{~B}_{1}(\sigma)$ state shown in Fig. 7 (TS1), which initiates the decay to COT $^{++}$.

In the third excited state the $\mathrm{b}_{2}$ SOMO is also localized in the central cyclobutane moiety, but the constituent AOs are aligned in the same directions as those in the $\mathrm{a}_{1}-\mathrm{MO}$, which results in a much better overlap of the two MOs. Hence the derivative coupling vector, which points also to an antisymmetric deformation of the fourmembered ring, is much longer than for the $a_{1}\left(\pi_{+}\right) / b_{1}(\sigma)$ interaction. In spite of the larger energy gap, the vibronic coupling between the ${ }^{2} A_{1}\left(\pi^{+}\right)$and the ${ }^{2} B_{2}(\sigma)$ state is therefore strong enough to overcome the force opposing the antisymmetric distortion of the central cyclobutane moiety, which makes that the potential curve along this coordinate bends down to form a double minimum (for the spontaneous cleavage of one or the other peripheral bond of the central four-membered ring).

TOD $^{*+}$ shows in fact a rather unusual case of vibronic coupling, because it is the third excited state which introduces a distortion of the ground state to cleave a bond that is rather sturdy in neutral TOD (it takes $c a$. $45 \mathrm{kcal} / \mathrm{mol}$ to form the biradical that corresponds to $\mathbf{B C B})$. Nevertheless it is a very good example to illustrate the workings of vibronic coupling, including the importance of the three factors that determine it.

The above explanations allow us to understand why TOD decays to $\mathbf{B C B}^{+}$and COT $^{++}$on ionization at $30 \mathrm{~K}$, but they do not explain the surprising formation of $\mathbf{T C O}^{*+}$ from $\mathbf{B C B}^{++}$, and its final decay to BOT $^{\circ+}$ (which we expected to be the primary product of the decay of $\mathbf{T O D}^{++}$!).

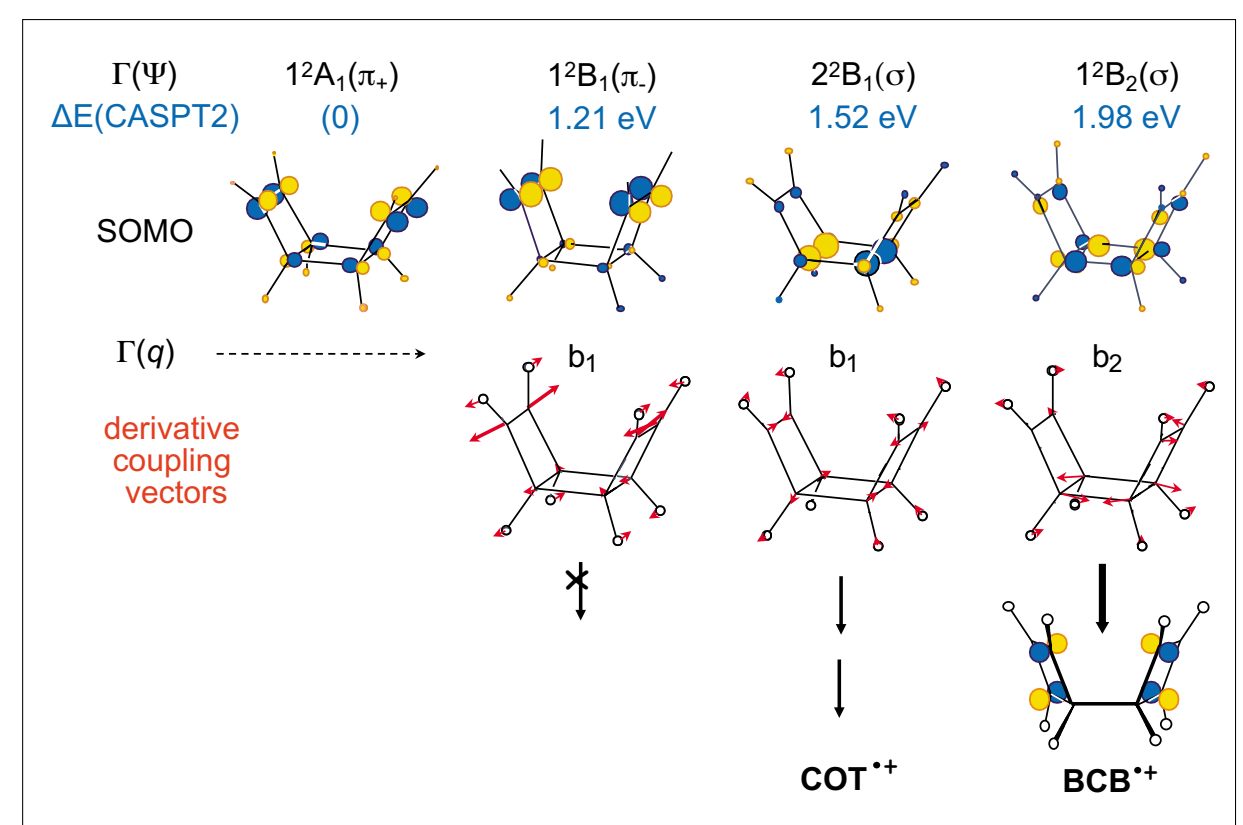

Fig. 7. The first three excited states of TOD॰+, characterized by their singly occupied MOs (SOMOs), and how they mix with the ground state along the deformations along the derivative coupling vectors. Discussion see text. 
One day Stephan Matzinger, one of my students, told me that that the unrestricted Hartree-Fock (UHF) wavefunction for $\mathbf{B C B}^{\cdot+}$ in $C_{2 v}$ symmetry is unstable, and that, if one reoptimizes that wavefunction without any constraints, it distorts spontaneously by localizing the spin mostly on one of the two cyclobutenyl moieties (and the charge mostly on the other), a process which leads to an energy lowering of 0.4 $\mathrm{kcal} / \mathrm{mol}$. If one reoptimizes the structure in the new $C$ symmetry, the charge-bearing moiety puckers (as it does in the cyclobutenium cation), the localization of the spin becomes even more pronounced (see Fig. 8 ), and the energy decreases by another $2.2 \mathrm{kcal} / \mathrm{mol}$. The resulting structure turns out to be a saddle point with an imaginary normal mode for twisting around the central $\mathrm{C}-\mathrm{C}$ bond. If one follows that mode one arrives at the minimum depicted on the right hand side of Fig. 8, which is another $2.5 \mathrm{kcal} / \mathrm{mol}$ more stable than the $C$ structure, presumably due to smaller steric repulsion.

If one does single-point DFT calculations at the three points shown in Fig. 8 (cf. last line), one finds that the process of spin localization is endothermic at this level, because electron correlation, which is not accounted for at the UHF level, favors delocalized structures (a bit too much at the DFT level, but that is another matter $\left.{ }^{[8]}\right)$. However, the question still poses itself what drives this localization, in the absence of electron correlation. The answer is that, once more, vibronic coupling is at work, this time between the ${ }^{2} \mathrm{~B}_{1}$ ground state and the ${ }^{2} \mathrm{~A}_{2}$ excited state of $\mathbf{B C B}^{\cdot+}$ ( $c f$. Fig. 4). This coupling is induced by $a b_{2}$ mode which results in a loss of the $C_{2}$ axis and the symmetry plane between the two cyclobutenyls and thus allows for a localization of the spin in one of the two allylic moieties, and for the ensuing puckering in $C_{\mathrm{S}}$ symmetry which leads to the structure shown in the middle of Fig. 8. The final twisting around the central $\mathrm{C}-\mathrm{C}$ bond is exothermic both at the UHF and the DFT levels of theory.

It was, however, the calculations at the UHF level of theory that led us to the solution of the puzzling question which of the $21 \mathrm{C}_{8} \mathrm{H}_{8}$ valence isomers ${ }^{[9]}$ was formed during the decay of $\mathbf{B C B}^{++}$and manifested itself by the broad band peaking at $820 \mathrm{~nm}$ (cf. Fig. 1). A DFT frequency calculation at the UHF minimum depicted on the right hand side of Fig. 8 revealed one imaginary mode, which we took as an invitation to see whether that structure happens to be close to a transition state. This turned out to be the case (TS3 in Fig. 9), and a forward IRC calculation showed the formation of a new bond between the terminus of allyl radical and the central atom of the allyl cation moiety (see dashed line in Fig. 8). During

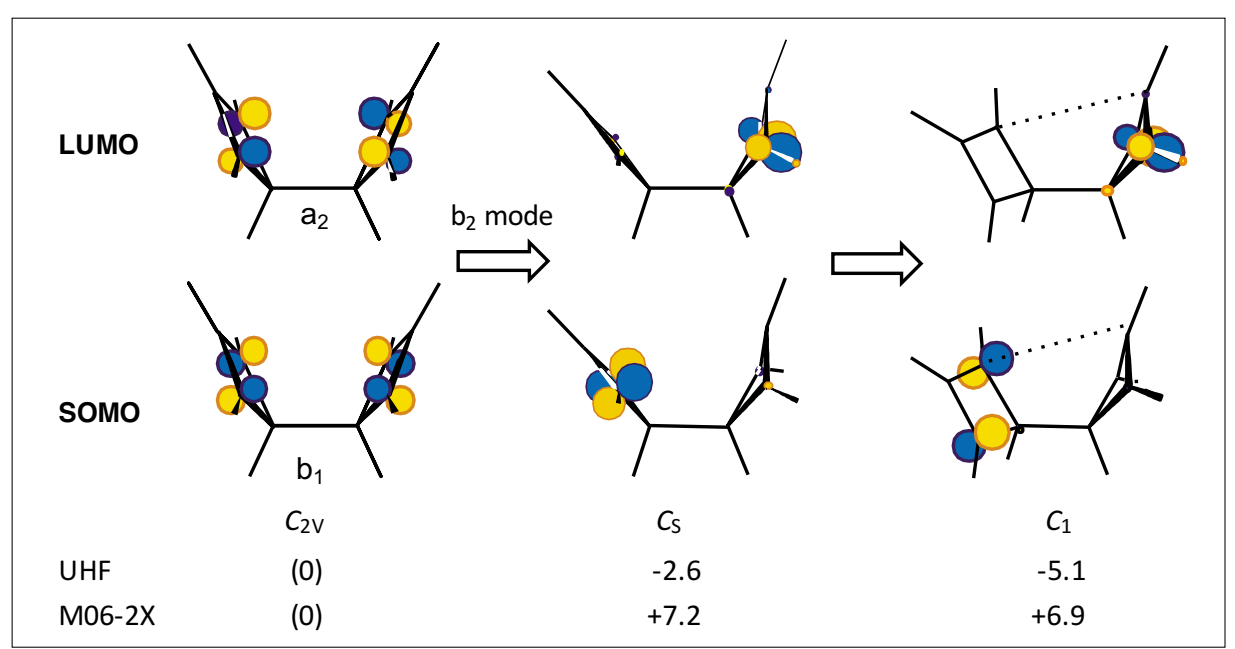

Fig. 8. spontaneous relaxation of $\mathbf{B C B}^{-+}$at the UHF level of theory. The dashed line indicates the new bond that is formed following this relaxation. Discussion see text.

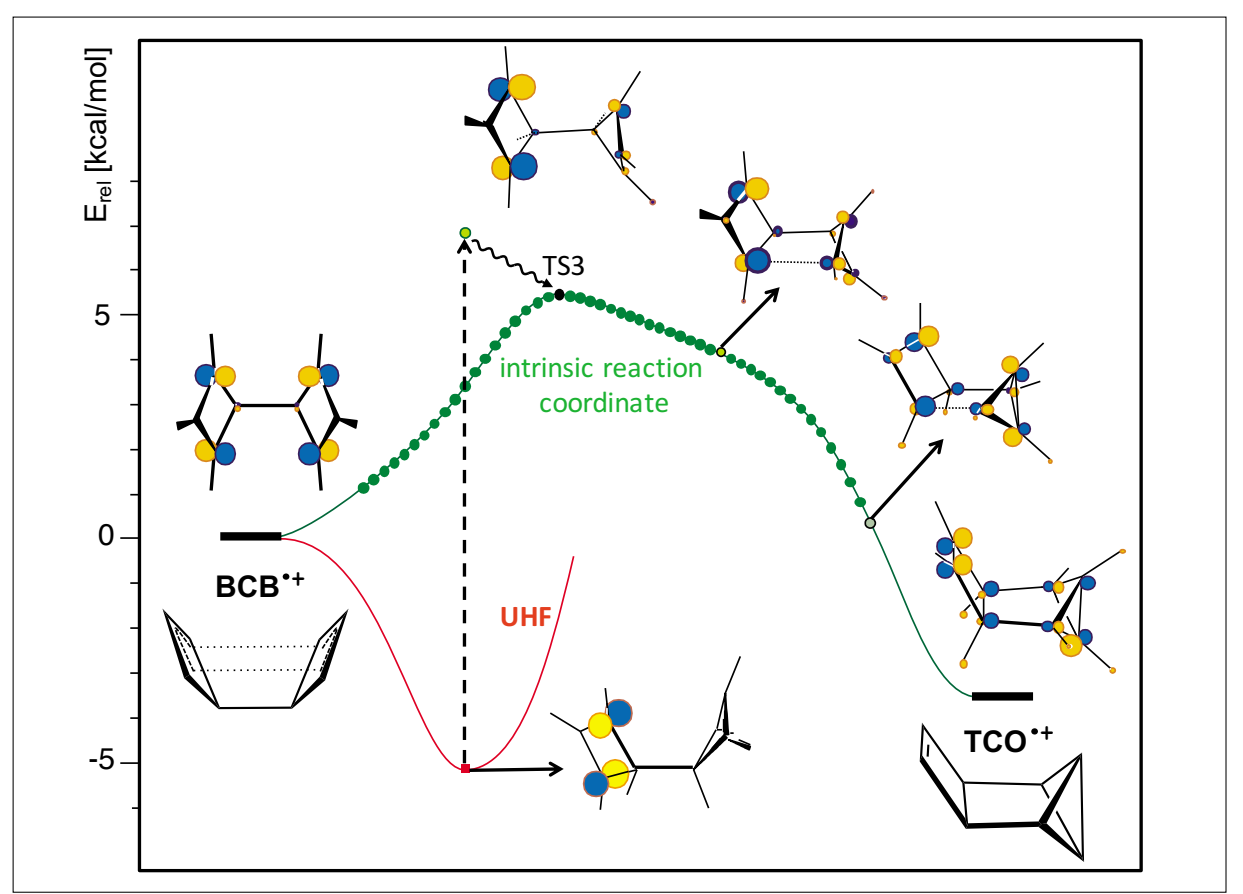

Fig. 9. Potential curve for the conversion of $\mathbf{B C B}^{*+}$ to $\mathbf{T C O}^{*+}$ (the IRC calculations were carried out at the B3LYP level of DFT theory).

the formation of this bond the puckering of the cyclobutenyl cation part of the molecule increased to the point where in the end it became a bicyclobutyl moiety, as it is the case in the final product, $\mathbf{T C O}^{*+}$, which, at the CCSD(T)// M06-2X level of theory is $9.2 \mathrm{kcal} / \mathrm{mol}$ more stable than $\mathbf{B C B}^{*+}$.

The activation barrier for the rearrangement of $\mathbf{B C B}^{++}$to $\mathbf{~ T C O}^{++}$is $5.4 \mathrm{kcal} / \mathrm{mol}$ at the M06-2X level of DFT theory and 6.8 $\mathrm{kcal} / \mathrm{mol}$ by the much more sophisticated $\operatorname{CCSD}(\mathrm{T})$ method, but if one accounts for the differences of zero-point energies, this number decreases by $c a$. $1 \mathrm{kcal} / \mathrm{mol}$, and consideration of the enthalpy corrections reduces it even a little more and brings it back into a range where it is entirely compatible with the observed slow unimolecular thermal rearrangement at $77 \mathrm{~K}$. The fact that the barrier for this rearrangement is so low is due to the vibronic coupling effects discussed above. to synthesize TCO, which can be made in four steps from benzvalene, ${ }^{[10]}$ and subject it to radiolysis at $77 \mathrm{~K}$. To our great satisfaction, the spectrum thus obtained ( $c f$. red trace in Fig. 1) turned out to be identical to that of the radical cation obtained upon the slow decay of $\mathbf{B C B}^{\circ+}$ at $77 \mathrm{~K}$ (blue trace in Fig. 1), so the problem, which had actually persisted for several years, was solved.

As shown in Fig. 3, TCO$^{-+}$is stable at $77 \mathrm{~K}$, but slowly rearranges to $\mathbf{B O T}^{++}$if one raises the temperature to $90 \mathrm{~K}$. We also did calculations on this reaction, which is exothermic by nearly $40 \mathrm{kcal} / \mathrm{mol}$. These calculations indicated that this decay is initiated by cleavage of one of the lateral bicyclobutyl bonds. However, in this case
With this finding in hand, we decided 


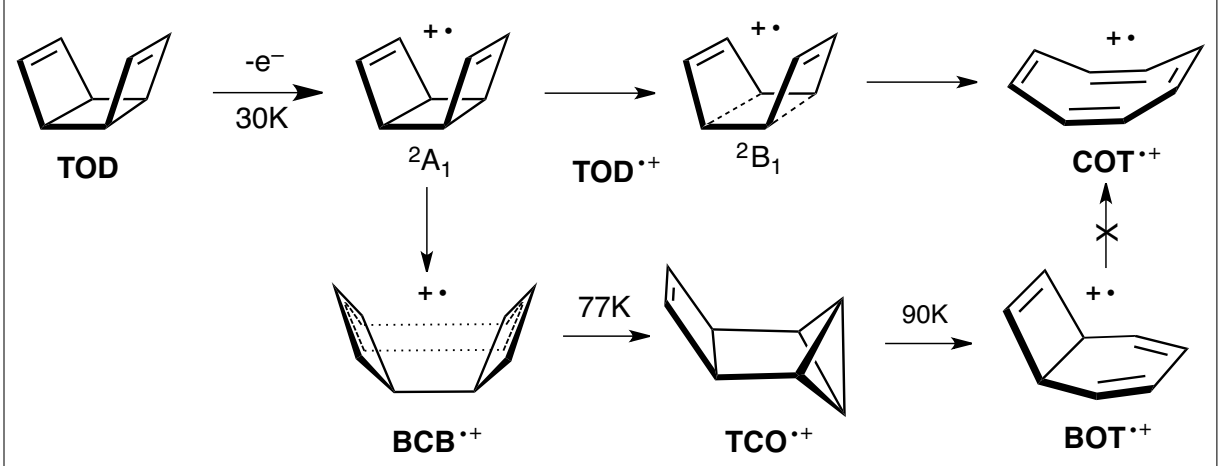

Scheme 2. The decay of TOD on ionization.

the calculated activation energy is much too high to be surmounted at $77 \mathrm{~K}(15.3$ $\mathrm{kcal} / \mathrm{mol}$ at the CCSD(T)//M06-2X level), which seems to indicate that there must be another mechanism leading to $\mathbf{B O T}^{\circ+}$, because it is very unlikely that calculations at this level of theory are off by almost 10 $\mathrm{kcal} / \mathrm{mol}$ when calculating activation barriers. As the experiments left no doubt that this rearrangement occurs indeed, we did not need a computational confirmation at that time, and we did not go to the trouble of systematically searching the potential energy surface for another pathway.

In sum, the decay of the radical cation of TOD and the intermediates formed during this process, which is summarized in Scheme 2, are excellent examples to illustrate how vibronic coupling to low-lying excited states makes that bonds that are rather strong in the neutral compounds cleave spontaneously on ionization, and why distortions caused by vibronic coupling can lead to rearrangements as surprising as the $\mathbf{B C B}^{\cdot+} \rightarrow \mathbf{T C O}^{\cdot+}$ reaction. This case also allows to illustrate the importance of state crossings in radical cations, which can prevent reactions that occur quite easily in neutrals (such as the electrocyclic $\mathbf{B C B}^{++} \rightarrow \mathrm{COT}^{*+}$ rearrangement) to have rather high barriers in radical cations. We will see more of this in the following two sections.

\section{The Case of the [1.1.1]Propellane Radical Cation[1b]}

[1.1.1]propellane (PRP) is the hydrocarbon with the highest strain energy per $\mathrm{C}-\mathrm{C}$ bond known. ${ }^{[11]}$ Nevertheless it is surprisingly stable, and, under exclusion of oxygen, it can be kept as a clear liquid at room temperature. We also found that, under flash vacuum pyrolysis conditions, it can be heated to $400{ }^{\circ} \mathrm{C}$ without decomposition. The high thermal stability has been attributed to the clasping effect of the three methylene groups which make that cleavage of the central $\mathrm{C}-\mathrm{C}$ bond to yield a very reactive biradical brings very little strain relief. ${ }^{[12]}$ The only viable alternative for PRP to get rid of some of its strain is to cleave one of the lateral bonds, to yield a methylene-bicyclobutyl biradical (MBC). Calculations indicate, however, that this biradical decays spontaneously by cleavshell singlet) methylene-cyclobutylidene carbene, a process that is still endothermic by $c a .45 \mathrm{kcal} / \mathrm{mol}$.

As PRP can be made in a single step from a precursor $(1,1$, -dibromo-2,2bis(chloromethyl)cyclopropane) ${ }^{[13]}$ which, at the time, was available from Aldrich, we decided to make this compound so we could look and see what would happen on removing an electron from it. Actually, we ing the central C-C bond to yield a (closed-

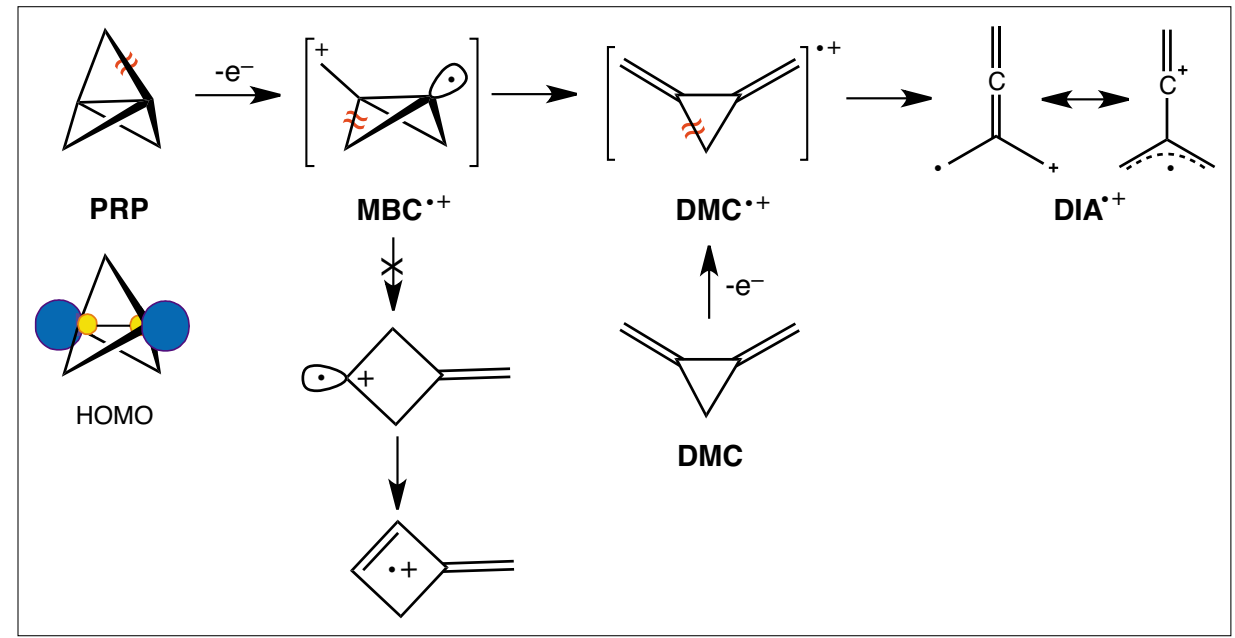

Scheme 3. Decay of [1.1.1]propellane on ionization.

expected that nothing would happen which, in view of the general lability of small-ring compounds on oxidation, would have been a surprise in and by itself. The reason for this expectation was, that the HOMO of PRP is almost nonbonding (see Scheme 3), so to remove an electron from this MO should have only minor structural consequences. In addition, the HOMO is totally symmetric, so the radical cation is not subject to Jahn-Teller distortion, in spite of its threefold symmetry.

However, it turned out that we were totally wrong, because on ionization of PRP not one but three of the lateral bonds cleave spontaneously to yield a species which can be regarded as an allyl radical joined with a vinyl cation, or a dimethyleneallene radical cation $\left(\mathbf{D I A}^{*+}\right)$, while the central bond of PRP remains intact! The spectroscopic data that led us to this conclusions can be found in the original publication. ${ }^{[1 b]}$

Of course we were flabbergasted by this finding, and, as in the above case of TOD, we wanted to know why a bond which takes $45 \mathrm{kcal} / \mathrm{mol}$ to break in the neutral molecule cleaves spontaneously in the radical cation. Again it turned out that vibronic coupling is responsible for this effect. This time the analysis was more difficult than in the case of TOD, because the first two excited states of $\mathbf{P R P}^{\boldsymbol{P}^{+}}$are degenerate. As a consequence, vibronic interaction with the two components of the first excited state does not lead to a double minimum, but to a minimum and a transition state which lie, however, very close in energy. But the minimum, where one of the lateral bonds in PRP is lengthened by $0.1 \AA$, turns out to be very shallow and stretching this bond by another $0.2 \AA$ leads to a transition state for the cleavage of that bond, which lies only $1.2 \mathrm{kcal} / \mathrm{mol}$ above PRP ${ }^{*+}$.

The resulting product, $\mathbf{M B C}^{\cdot+}$, which is $7.4 \mathrm{kcal} / \mathrm{mol}$ more stable than $\mathbf{P R P}^{++}$, is a minimum on the potential surface, in contrast to the neutral biradical, where the central bond cleaves spontaneously. Thus one would expect that the central bond of MBC $^{++}$is the most labile one, because its cleavage would lead to the ionized methylene-cyclobutylidene carbene. However, this species lies above $\mathbf{M B C}^{\circ+}$ and undergoes a spontaneous $\mathrm{H}$-shift to yield the much more stable radical cation of methylenecyclobutene (see Scheme 3). Instead, one of the lateral bonds of $\mathbf{M B C}^{\cdot+}$ breaks, with a barrier of only $1.1 \mathrm{kcal} / \mathrm{mol}$ to form the radical cation of dimethylenecyclopropene $\left(\mathbf{D M C}^{\circ+}\right)$ which is $c a .17 \mathrm{kcal} / \mathrm{mol}$ more stable than $\mathbf{M B C}^{\circ+}$. This cleavage is also driven by vibronic interaction between the symmetric ground state of $\mathbf{M B C}{ }^{\circ+}$ with an antisymmetric low-lying excited state.

Another big surprise was that the decay of $\mathbf{P R P}^{*+}$ does not end with $\mathbf{D M C}^{\cdot+}$, 
which looks like a perfectly stable radical cation, because an electron is missing from the $\pi$-system, which usually has no big structural consequences. In fact, when one ionizes $\mathbf{D M C}^{\cdot+}$ it also decays spontaneously to DIA*+ (and to vinylallene, but that is another story). Calculations showed that the barrier for cleavage of one of the lateral bonds involves once more a barrier of only $1.7 \mathrm{kcal} / \mathrm{mol}$. Again, the surprising weakness of those lateral bonds is caused by vibronic coupling, between the ${ }^{2} \mathrm{~A}$, ground state and the ${ }^{2} \mathrm{~B}_{1}$ excited state of DMC $^{\cdot+}$ which, according to photoelectron spectroscopy, are separated by only $1.4 \mathrm{eV}$.

The mode that couples these two states must therefore have $b_{2}$ symmetry which might imply an antisymmetric deformation of the three-membered ring. However, the derivative coupling vector coincides with the antisymmetric stretching mode of the two double bonds (which is indeed lowered by $c a .400 \mathrm{~cm}^{-1}$ on going from neutral DMC to its radical cation), a mode which has a high force constant and cannot lead to any changes in the bonding mode of DMC $^{\bullet+}$. However, following the $b_{2}$ mode that implies an antisymmetric stretching of the two lateral $\sigma$-bonds (the frequency of which is lowered by only $30 \mathrm{~cm}^{-1}$ on going from the neutral to the cation) leads to the very low-lying transition state for the formation of $\mathbf{D I A}{ }^{\cdot+}$. Apparently, the vibronic coupling becomes stronger as one proceeds along this mode, and as the force constant of the stretched bond diminishes, this is what leads to the easy cleavage of that bond.

Thus, all three processes shown in Scheme 3 are driven by vibronic coupling, which makes [1.1.1]propellane another stunning example of the importance of this effect in radical cations.

\section{The Ring Opening of the Cyclo- butene Radical Cation (CB $\left.{ }^{\bullet+}\right)^{[14]}$}

During the first years of chemistry we learn that the ring-opening of cyclobutene is a textbook example for the operation of the Woodward-Hoffman rules for electrocyclic reactions. We wanted to investigate whether similar rules can also be applied to radical cations, but we found that this is not the case, because ionization of $\mathbf{C B}$ in $\mathrm{Ar}$ matrices led directly to the trans isomer of the butadiene radical cation $\left(\mathbf{B D}^{++}\right)$, while no trace of the $c i$-isomer was ever seen. ${ }^{[1 \mathrm{c}]}$ Hence the ring-opening of $\mathbf{C B}^{\cdot+}$ cannot be governed by orbital (or state) symmetry rules, as it is the case for the neutral reaction.

The reason is that electrocyclic reactions of radical cations usually involve crossings of states of different symmetry, if a symmetry element is maintained along

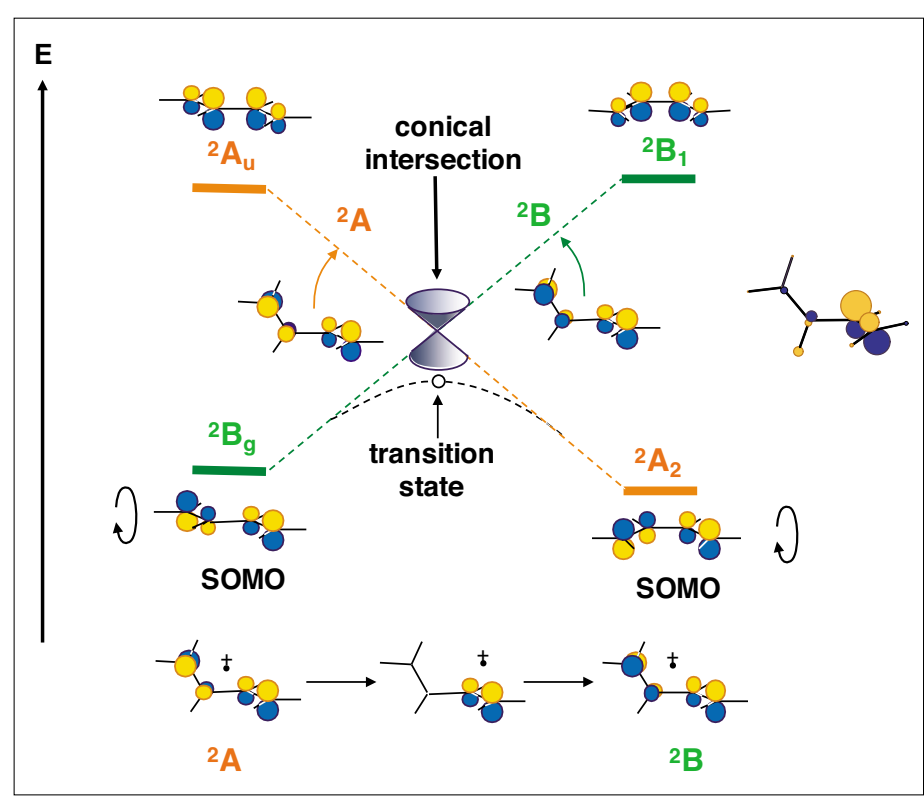

Fig. 10. State correlation diagram for the cis-trans isomerization of BD*+ the reaction path (as it is done if one applies the Woodward-Hoffmann rules). This feature can be conveniently illustrated by the cis-trans-isomerization of the radical cation of butadiene $\left(\mathbf{B D}^{-+}\right)$, shown in Fig. 10.

In the ground state of $\mathbf{B D}^{\mathbf{*}}$ the SOMO is the $\pi_{2}$ MO which has a node in the $\pi$-system ( $b_{\mathrm{g}}$ in the case of the trans-, $\mathrm{a}_{2}$ in the case of the cis-isomer). If one twists around the central bond of either isomer, the SOMO turns into the $\pi_{1} \mathrm{MO}$, which has no node. Thus, there is one electron in $\pi_{1}$ and two in the higher-lying $\pi_{2} \mathrm{MO}$, which implies that, in the course of this isomerization, one has landed in an excited state of $\mathbf{B D}^{\cdot+}$. Neutral electrocyclic reactions where the ground state correlates with a (doubly) excited state are called 'forbidden', which means that their activation energy is higher than that for the analogous 'allowed' process (where the ground states of the reactants and products correlate).

In radicals, 'allowed' isomerizations are rare, and state crossings are ubiquitous if a symmetry element is retained in the process. What happens along such reaction paths is that the ground and the excited state approach each other on the way to the state crossing (which is called 'conical intersection'). As the energy difference be-

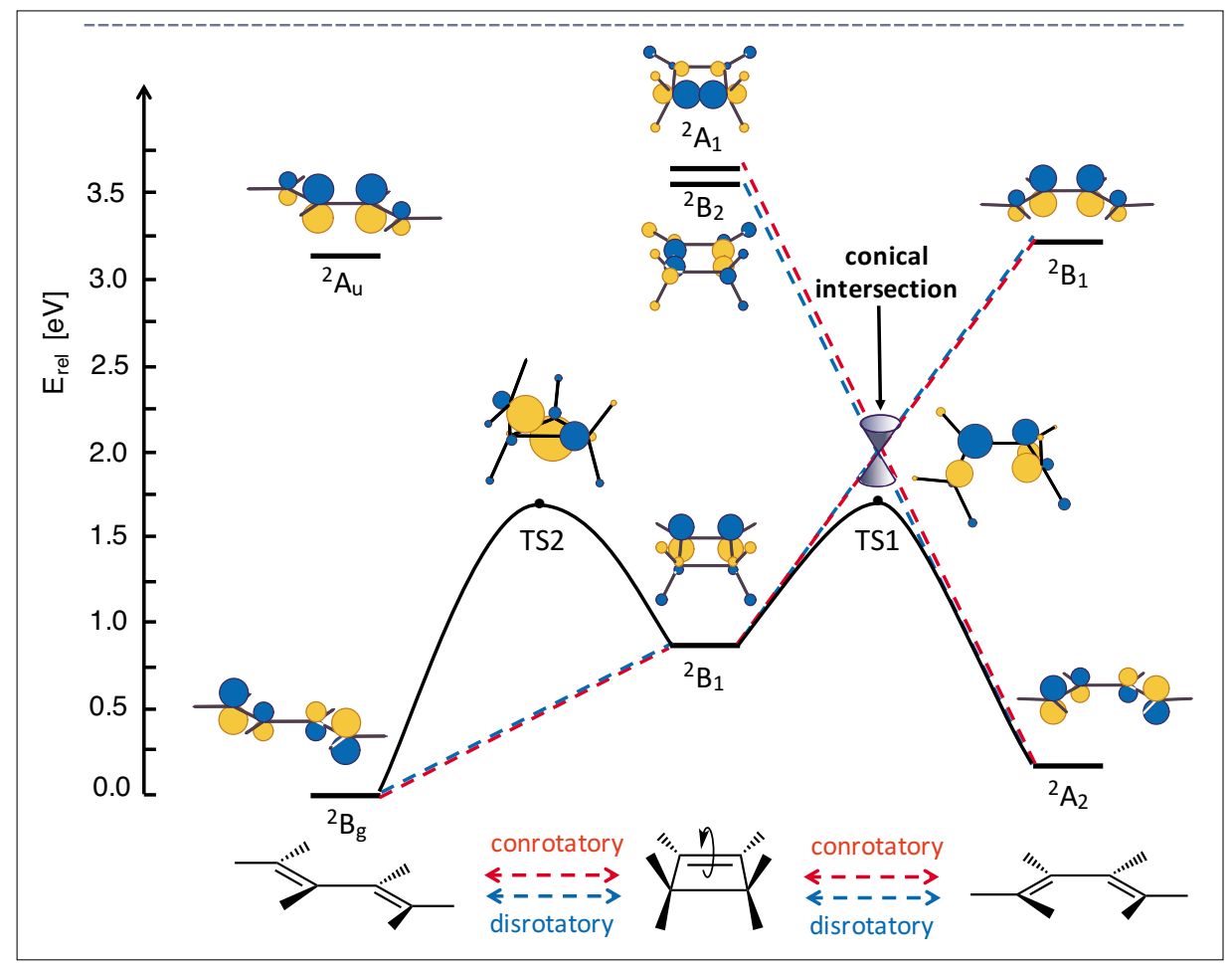

Fig. 11. State correlation diagram for the con- or disrotatory ring opening of $\mathbf{C B}^{\bullet+}$ to cis- or transBD ${ }^{+}$. Relative energies are from (TD)DFT calculations. The different states of the three radical cations are characterized by their SOMOs. 
tween the two states diminishes, vibronic coupling between them increases ( $c f$. Eqn. (1)), along a coordinate that breaks the symmetry (in the case illustrated in Fig. 10 this is a $C_{2}$ axis). This distortion allows the system to circumvent the conical intersection and find a lower energy pathway around it, on which it passes through a transition state.

In the case of the reaction illustrated in Fig. 10, an important feature of the transit from the orange ${ }^{2} \mathrm{~A}$ to the green ${ }^{2} \mathrm{~B}$ surface is, that the spin and charge must localize in one of the two double bonds of $\mathbf{B D}^{++}$ so the coefficients of the SOMO can reemerge with the opposite sign, as shown at the bottom of Fig. 10. This localization creates difficulties in calculations with DFT methods which are reluctant to localize unpaired electrons (due to the incomplete cancellation of the self-repulsion of these electrons). ${ }^{[8]}$ At the highest level of theory which we employed, the activation barrier for rotation around the central bond in $\mathbf{B D}^{\cdot+}$ is $28 \mathrm{kcal} / \mathrm{mol}$, compared to $c a .3$ $\mathrm{kcal} / \mathrm{mol}$ in neutral BD. ${ }^{[15]}$

But let us return to the cyclobutene ringopening, the state-correlation diagram for which is shown in Fig. 11.[14] On the right hand side of this Figure one can see that, if the radical cation of cyclobutene $\left(\mathbf{C B}^{\cdot+}\right)$ undergoes electrocyclic ring-opening to cis-BD ${ }^{\bullet+}$ in the con- or disrotatory fashion, while an axis or a plane of symmetry is retained, respectively, the ground states of the two compounds invariably correlate with excited states of the products. Thus the systems evolve towards conical intersections which are avoided by vibronic coupling which breaks the remaining symmetry. Indeed a transition state, TS1, can be found for the conrotatory ring opening which lies ca. $18 \mathrm{kcal} / \mathrm{mol}$ above $\mathbf{C B}^{\cdot+}$ at the CCSD(T)//M06-2X level.

The left hand side of Fig. 11 shows, however, that if one proceeds from $\mathbf{C B}^{\cdot+}$ to trans $-\mathbf{B D}^{\circ+}$ by twisting around the $\pi$-bond of $\mathbf{C B}^{\cdot+}$, the ground states of the two compounds correlate adiabatically, i.e. this reaction is symmetry-allowed! However, proceeding along this reaction coordinate requires a lot of energy because two bonds must be broken at the same time (although the $\pi$-bond of $\mathbf{C B}^{\cdot+}$ is only half a double bond, $c f$. SOMO).

As a consequence, the activation barrier for this process was found to be very similar to that for the 'normal' ring-opening to cis-BD', i.e. also $18 \mathrm{kcal} / \mathrm{mol}$. However, in spite of the fact that the conrotatory reaction could in principle follow a path of $C_{2}$ symmetry without encountering any conical intersections, only a second-order saddle point was found along this coordinate, which distorted to a true transition state, TS2, under the impact of vibronic coupling to a very low-lying excited state of opposite symmetry than the ground state. In fact, TS2, turned out to be completely non-symmetric and it reminded us of a cyclopropyl-carbinyl intermediate that Nate Bauld had postulated to occur during this reaction in the 1980s.

\section{Conclusion}

In this article, I have used three examples from our research to illustrate the importance of vibronic coupling between ground and excited states - a concept that is rarely considered in the chemistry of closed-shell species - in reactions of radical cations. The main reason why vibronic coupling manifests itself more pronouncedly in radical cations is because its extent is inversely proportional to the energy difference between two states. Since radical cations often have low-lying excited states (radical cations of neutral compounds which absorb only in the UV are often deeply colored!), they are more susceptible to show the effects of vibronic coupling. However, the example of TOD ${ }^{++}$ shows also, that this is not the only factor that determines the 'chemical' consequences of vibronic coupling: the overlap of the two singly occupied MOs of two radical cation states determines the length of the derivative coupling vector which indicates the direction of the distortion which optimally mixes the two states, and the force constant that opposes this distortion determines whether vibronic coupling manages to deform the potential surface along this coordinate such that bonds break spontaneously or not. The latter two factors make that it is in fact the third excited state that induces a distortion that leads to a bond cleavage in TOD ${ }^{+}$!

However, where the energy difference between two states becomes decisive is when, in the course of a chemical reaction, a molecule proceeds towards a state crossing (called 'conical intersection'), as illustrated in the last case. Thereby the energy differences between the ground and the excited states diminishes continuously and, as the system proceeds along the symmetry-restrained reaction path (e.g. con- or disrotatory ring opening), vibronic coupling invariably becomes strong enough that it forces the molecule to deviate from that path and circumvents the conical intersection, thus lowering the barrier for the reaction. Nevertheless, calculations showed that the barrier for the 'electrocyclic' ring opening of $\mathbf{C B}^{\cdot+}$ is so high that the process that leads directly to trans-BD+ ${ }^{++}$becomes competitive, and wins in reality.

Electrocylic reactions of radical cations are an interesting field of research full of surprises where the rules we have learned to apply to the corresponding neutral re- actions do not apply, and are replaced by other factors, the most important of which is often vibronic coupling to low-lying excited states. In the course of our research we have learned to predict the effects of vibronic coupling, qualitatively and on the basis of quantum chemical calculations.

\section{Acknowledgements}

I would like to express my sincere gratitude to the graduate students and postdocs who participated with great enthusiasm, patience and persistency in the studies that are described in this paper, and to our colleagues at other universities who often contributed essential experimental results to resolve the questions that were posed by our experiments and calculations. I also want to thank the Swiss National Science Foundation for its unfailing support of our research, in spite of the fact that it often took a very different direction than what we had proposed.

Received: December 5, 2015

[1] a) T. Bally, S. Bernhard, S. Matzinger, J.-L. Roulin, G. N. Sastry, L. Truttmann, Z. Zhu, A. Marcinek, J. Adamus, R. Kaminski, J. Gebicki, F. Williams, G.-F. Chen, M. Fülscher, Chem. Eur. J. 2000, 6, 858; b) B. Müller, T. Bally, R. Pappas, F. Williams, J. Am. Chem. Soc. 2010, 132, 14649; c) J.-N. Aebischer, T. Bally, K. Roth, E. Haselbach, F. Gerson, S. Z. Qin, J. Am. Chem. Soc. 1989, 111, 7909.

[2] T. Bally, 'Electronic Structure, Spectroscopy and Photochemistry of Organic Radical Cations', in 'Radical Ionic Systems, Properties in Condensed Phase', Eds. A. Lund, M. Shiotani, Kluwer: Dordrecht, 1991, pp 3.

[3] H. M. Frey, H.-D. Martin, M. Hekman, J. Chem. Soc. Chem. Comm. 1975, 204.

[4] R. Gleiter, E. Heilbronner, H. Mirko, H.-D. Martin, Chem. Ber. 1973, 106, 28.

[5] a) B. Badger, B. Brocklehurst, Nature (London) 1968, 219; b) B. Badger, B. Brocklehurst, Trans. Faraday Soc. 1970, 2939.

[6] B. K. Carpenter, 'Potential energy surfaces and reaction dynamics', in 'Reactive Intermediate Chemistry', Eds. R. A. Moss, M. S. Platz, M. Jones, Wiley: Hoboken, NJ, 2005, pp 925.

[7] L. Köppel, L. S. Cederbaum, W. Domcke, S. Shaik, Angew. Chem. Int. Ed. Engl. 1983, 22, 210.

[8] T. Bally, G. N. Sastry, J. Phys. Chem. A 1997, 101, 7923.

[9] a) L. R. Smith, J. Chem. Educ. 1978, 55, 569; b) K. Hassenrück, H.-D. Martin, R. Walsh, Chem. Rev. 1989, 89, 1125.

[10] L. R. Smith, G. E. Gream, J. Meinwald, J. Org. Chem. 1977, 42, 927.

[11] K. B. Wiberg, Angew. Chem. Int. Ed. 1986, 25, 312 .

[12] K. B. Wiberg, Chem. Rev. 1989, 89, 975.

[13] J. Belzner, U. Bunz, K. Semmler, G. Szeimies, K. Opitz, A.-D. Schlüter, Chem. Ber. 1989, 122, 397.

[14] G. N. Sastry, T. Bally, V. Hrouda, P. Cársky, J. Am. Chem. Soc. 1998, 120, 9323.

[15] One can also say that this is due to the fact that an electron is ejected from the HOMO of BD that is antibonding along the central bond, which makes that upon ionization the central bond becomes a partial double bond. 